\title{
Retrospective Molecular Epidemiology Study of PD-L1 Expression in Patients with EGFR-Mutant Non-small Cell Lung Cancer
}

\author{
Jong Ho Cho, MD, PhD' \\ Wei Zhou, PhD2 \\ Yoon-La Choi, MD, PhD 3 \\ Jong-Mu Sun, MD, PhD ${ }^{4}$ \\ Hyejoo Choi, BSc ${ }^{1}$ \\ Tae-Eun Kim, $\mathrm{RN}^{1}$ \\ Marisa Dolled-Filhart, $\mathrm{PhD}^{2}$ \\ Kenneth Emancipator, $\mathrm{MD}^{2}$ \\ Mary Anne Rutkowski, MS² \\ Jhingook Kim, MD, PhD'
}

\begin{abstract}
Purpose
Data are limited on programmed death ligand 1 (PD-L1) expression in epidermal growth factor receptor (EGFR)-mutant non-small cell lung cancer (NSCLC).

\section{Materials and Methods}

We retrospectively evaluated the relationship between PD-L1 expression and recurrencefree survival (RFS) and overall survival in 319 patients with EGFR-mutant NSCLC who were treated at Samsung Medical Center from 2006 to 2014. Membranous PD-L1 expression on tumor cells was measured using the PD-L1 IHC 22C3 pharmDx antibody and reported as tumor proportion score (TPS). Kaplan-Meier methods, log-rank test, and Cox proportional hazards models were used for survival analysis.
\end{abstract}

\section{Results}

All patients had $\geq 1$ EGFR mutation-54\% in exon 19 and $39 \%$ in exon 21 . Overall, $51 \%$ of patients had PD-L1-positive tumors. The prevalence of PD-L1 positivity was higher among patients with stages II-IV versus stage I disease (64\% vs. $44 \%$ ) and among patients with other EGFR mutations (75\%) than with L858R mutation (39\%) or exon 19 deletion (52\%). PD-L1 positivity was associated with shorter RFS, with an adjusted hazard ratio of 1.52 (95\% confidence interval [Cl], 0.81 to 2.84; median, 18 months) for the PD-L1 TPS $\geq 50 \%$ group, 1.51 (95\% Cl, 1.02 to 2.21; median, 31 months) for the PD-L1 TPS 1\%-49\% group, and 1.51 (95\% Cl, 1.05 to 2.18) for the combined PD-L1-positive groups (TPS $\geq 1 \%$ ) compared with the PD-L1-negative group (median, 35 months).

\section{Conclusion}

PD-L1 expression is associated with disease stage and type of EGFR mutation. PD-L1 positivity might be associated with worse RFS among patients with surgically treated EGFRmutant NSCLC.
Correspondence: Jhingook Kim, MD, PhD Department of Thoracic Surgery, Samsung Medical Center, Sungkyunkwan University School of Medicine, 81 Irwon-ro, Gangnam-gu, Seoul 06351, Korea

Tel: 82-2-3410-3483

Fax: 82-2-3410-6986

E-mail: jhingookkim@gmail.com

Received December 15, 2016

Accepted February 28, 2017

Published Online March 17, 2017

*Jong Ho Cho and Wei Zhou contributed equally to this work.

\section{Key words}

Programmed cell death 1 protein,

Epidermal growth factor receptor, Non-small cell lung carcinoma 


\section{Introduction}

Approximately $80 \%-85 \%$ of all lung cancers worldwide are non-small cell lung cancers (NSCLCs) [1]. There are three main subtypes of NSCLC, of which adenocarcinoma is the most frequent histologic subtype [2]. Though histologic subtype provides a basis for choice of chemotherapy, overall survival (OS) remained poor regardless of subtype of NSCLC $[3,4]$.

The emergence of targeted therapy for driver oncogenes involved in NSCLC revolutionized its treatment [3]. Early therapies targeted epidermal growth factor receptor (EGFR) or anaplastic lymphoma kinase $(A L K)$ mutations $[3,4]$. Patients without these oncogenes receive platinum-based chemotherapy as first-line therapy, whereas patients with activating EGFR mutations receive tyrosine kinase inhibitors (TKIs) as first-line therapy [1,3,4]. Among Asian patients with NSCLC, 30\%-51\% have EGFR mutations [2,5] compared with $<10 \%$ of non-Asian patients, which can improve the survival of Asian patients with advanced or recurrent NSCLC [2]. However, most patients who respond to these therapies eventually develop resistance and progress.

Some tumors, including NSCLC, have been shown to avoid destruction by the innate immune system by overexpression of programmed death 1 (PD-1) ligands [6-8]. Our previous results suggested that positive PD ligand 1 (PD-L1) expression may be a negative prognostic factor among patients with NSCLC who underwent surgery, most notably among patients with adenocarcinoma $[9,10]$. Thus, targets of the PD-1 pathway are an appealing option for potential therapeutics. Two humanized anti-PD-1 antibodies (nivolumab and pembrolizumab) [11,12] and 1 humanized anti-PD-L1 antibody (atezolizumab) [13] are currently approved in the United States for treatment of NSCLC, and several additional monoclonal antibodies targeting the PD-1/PD-L1 pathway are under investigation [10,14-17].

To date, there are limited data on PD-L1 expression in patients with EGFR-mutant NSCLC and whether the prevalence or the prognostic effect of PD-L1 expression may vary among different types of EGFR mutations. We evaluated the relationship between PD-L1 expression and clinical characteristics, including age, sex, smoking history, stage, and recurrence-free survival (RFS), and OS, among patients with surgically resected EGFR-mutant NSCLC, using the U.S. Food and Drug Administration (FDA)-approved companion diagnostic immunohistochemical assay PD-L1 IHC 22C3 pharmDx (Dako North America, Carpinteria, CA) [18,19].

\section{Materials and Methods}

\section{Study design and patients}

A total of 5,505 patients with NSCLC (2,815 of these patients with adenocarcinoma) underwent surgical resection at Samsung Medical Center (Seoul, Korea) between April 1, 2006, and January 31, 2014. Eligible patients in this study were those who had histologically confirmed NSCLC, confirmed EGFR mutation, sufficient tissue for PD-L1 immunohistochemical staining, and complete clinical and outcome information $(\mathrm{n}=323)$. EGFR mutation status was confirmed using direct sequencing or peptide nucleic acid-clamp method of exon 18 through 21 of chromosome 7 at Samsung Medical Center. Baseline demographics and disease characteristics (including age, sex, histologic type, stage, and Eastern Cooperative Oncology Group performance status) and clinical outcomes (including treatment and dates of diagnosis, surgery, and death or recurrence) were retrospectively obtained from medical records. Pathologic tumor stage was defined using the American Joint Committee on Cancer cancer staging manual, seventh edition [20]. Stage was assigned retrospectively for patients whose tumors were staged before publication of the seventh edition. Smoking status was defined as never ( $<100$ lifetime cigarettes) or current (quit $<1$ year before diagnosis). The study was approved by the appropriate institutional review board.

\section{PD-L1 immunohistochemistry}

PD-L1 expression was assessed in formalin-fixed paraffinembedded tumor samples acquired by surgical resection of each patient's individual sample, using the PD-L1 IHC 22C3 pharmDx assay (Dako North America). All PD-L1 assays were performed at LabCorp Clinical Trials, Los Angeles (Laboratory Corporation of America, Burlington, NC), the same laboratory, using the same procedures, that was employed in the pembrolizumab clinical trials for NSCLC $[21,22]$. The assay is described in detail elsewhere $[18,19]$. Membranous PD-L1 expression on tumor cells was defined by tumor proportion scores (TPSs) of $\geq 50 \%$ and $1 \%-49 \%$, respectively (Fig. 1), consistent with the cutoffs used in pembrolizumab clinical trials [21,22].

\section{Statistical methods}

The prevalence of PD-L1 TPS $\geq 50 \%$ and TPS $1 \%-49 \%$ was compared with the use of chi-square analysis in different subgroups based on age, sex, smoking status, stage, and types of EGFR mutations. RFS was defined as time from the date of diagnosis to the date of recurrence, death, or last fol- 

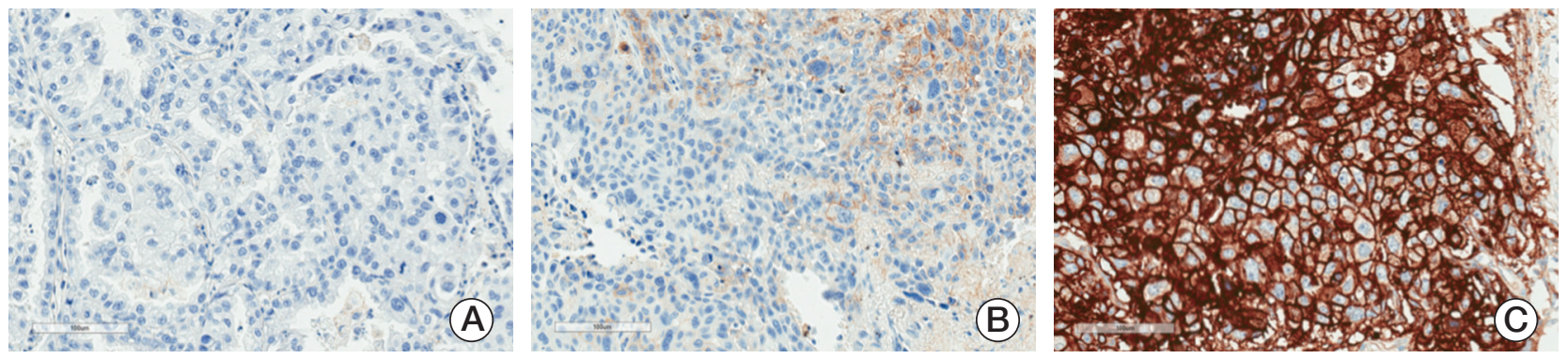

Fig. 1. Sample images of programmed death ligand 1 (PD-L1) staining in non-small cell lung cancer. (A) PD-L1 negative (tumor proportion score [TPS] < 1\%). (B) PD-L1 TPS 1\%-49\%. (C) PD-L1 TPS $\geq 50 \%$. All images are at original magnification $\times 20$, blue counterstain is hematoxylin, and PD-L1 is identified by brown chromagen.

low-up. OS was defined as time from the date of diagnosis to the date of death or last follow-up. Kaplan-Meier method, log-rank test, and Cox proportional hazards models were used to analyze the relationship between PD-L1 expression and RFS and OS, with the PD-L1-negative group as the reference group.

Two adjusted Cox proportional hazards models were used. In each model, the PD-L1-negative population was the reference population. The first Cox model adjusted for known baseline prognostic factors, including age, sex, smoking status, disease stage, and Eastern Cooperative Oncology Group performance status. The second Cox model adjusted for these known baseline prognostic factors, as well as postsurgical chemotherapy, including EGFR TKI and radiation therapy. Different types of EGFR mutations were also included in the initial models, and covariates included in the final models were based on a backward stepwise variable selection process. Statistical analyses were performed using SAS ver. 9.3 (SAS Institute Inc., Cary, NC); $\mathrm{p} \leq 0.05$ was considered to be statistically significant.

\section{Results}

\section{Patient population}

Among the 323 patients who were included in the original data set, PD-L1 expression status for four patients could not be evaluated because of insufficient tumor cells in the slides, leaving 319 patients in the final analysis. Among those, median age was 62.0 years (range, 35 to 84 years), 61\% were women $(n=194), 39 \%$ were men $(n=125), 64 \%$ were never smokers $(n=205)$, and $36 \%$ were smokers $(n=114)$. The majority of the patients $(n=311,97 \%)$ had adenocarcinoma, reflecting the clinical representation of EGFR mutations in Asian populations. Most patients had early-stage disease, including $49 \%$ $(\mathrm{n}=153)$ stage IA, 15\% $(\mathrm{n}=48)$ stage IB, 13\% $(\mathrm{n}=41)$ stage II, and $20 \%$ ( $n=61)$ stage III. Additionally, 5\% $(n=16)$ were stage IV.

\section{Treatment information}

All 319 patients received surgery, with 94\% of curative intent. Additionally, 30\% $(n=95)$ received postsurgery adjuvant chemotherapy including five patients had EGFR TKIs (mainly gefitinib for the palliative purpose due to pleural seeding during the surgery), and 10\% ( $\mathrm{n}=33)$ received adjuvant radiation. A total of 128 patients had recurrence, among whom $84 \%$ ( $n=108)$ received postrecurrence EGFR TKIs, and 59 of the 108 patients also received additional chemotherapy; 39\% of the 128 patients $(n=50)$ received postrecurrence radiation.

\section{EGFR, KRAS, and ALK status}

All 319 patients had tumors with at least one EGFR mutation (Table 1). Among the EGFR mutations, 171 patients $(54 \%)$ had a mutation in exon 19, including 145 with a deletion and others with complex mutations; 124 patients (39\%) had a mutation in exon 21, including 121 with an L858R mutation and three with other mutations; 12 patients $(4 \%)$ had a missense mutation in exon 18; and eight patients $(2 \%)$ had a mutation in exon 20 including insertion, deletion, and missense mutations. One patient had mutations in both exons 18 and 20, and three patients $(1 \%)$ had other mutations.

Among the 319 patients, 305 were also tested for Kirsten rat sarcoma (KRAS) mutation at exons 12, 13, and 61. Only one patient was confirmed as KRAS mutation positive at exon 13 with PD-L1 TPS of 1\%-49\%. Two-hundred eighty patients had $A L K$ status by immunohistochemistry; only two were $A L K$ 
Table 1. PD-L1 expression status by subgroup

\begin{tabular}{|c|c|c|c|c|c|}
\hline \multirow{2}{*}{ Subgroup } & \multirow{2}{*}{ No. } & \multicolumn{3}{|c|}{ PD-L1 expression status } & \multirow{2}{*}{$\begin{array}{l}\text { Chi-squar } \\
\text { p-value }\end{array}$} \\
\hline & & TPS $\geq 50 \%$ & TPS $1 \%-49 \%$ & Negative & \\
\hline Overall & 319 & $24(8)$ & $139(44)$ & $156(49)$ & \\
\hline \multicolumn{6}{|l|}{ Sex } \\
\hline Male & 125 & $13(10)$ & $64(51)$ & $48(38)$ & 0.008 \\
\hline Female & 194 & $11(6)$ & $75(39)$ & $108(56)$ & \\
\hline \multicolumn{6}{|l|}{ Smoking status } \\
\hline Never & 205 & $11(5)$ & $82(40)$ & $112(55)$ & 0.011 \\
\hline Smokers & 114 & $13(11)$ & $57(50)$ & $44(39)$ & \\
\hline \multicolumn{6}{|l|}{ ECOG PS } \\
\hline 0 & 202 & $12(6)$ & $88(44)$ & $102(51)$ & 0.345 \\
\hline 1 & 116 & $12(10)$ & $50(43)$ & $54(47)$ & \\
\hline \multicolumn{6}{|c|}{ Disease stage at baseline } \\
\hline IA & 153 & $6(4)$ & $61(40)$ & $86(56)$ & 0.006 \\
\hline IB & 48 & $1(2)$ & $20(42)$ & $27(56)$ & \\
\hline II & 41 & $7(17)$ & $20(49)$ & $14(34)$ & \\
\hline III & 61 & $9(15)$ & $29(48)$ & $23(38)$ & \\
\hline IV & 16 & $1(6)$ & $9(56)$ & $6(38)$ & \\
\hline \multicolumn{6}{|l|}{ EGFR mutation } \\
\hline Exon 19 deletion & 145 & $9(6)$ & $67(46)$ & $69(48)$ & 0.001 \\
\hline Exon 21 L858R & 121 & $9(7)$ & $38(31)$ & $74(61)$ & \\
\hline Others ${ }^{\text {a) }}$ & 53 & $6(11)$ & $34(64)$ & $13(25)$ & \\
\hline
\end{tabular}

Values are presented as number (\%). PD-L1, programmed death ligand 1; TPS, tumor proportion score; ECOG PS, Eastern Cooperative Oncology Group performance status; EGFR, epidermal growth factor receptor. ${ }^{\mathrm{a}}$ Exon 19, nondeletion $(\mathrm{n}=26)$; exon 21, non-L858R mutation ( $\mathrm{n}=3)$; exon $18(\mathrm{n}=12)$; exon $20(\mathrm{n}=8)$; one patient had mutations in both exons 18 and 20, and three patients had other mutations. Among those $(n=53)$, median age was 60.0 years (range, 44 to 84 years), $51 \%$ were women $(\mathrm{n}=27), 49 \%$ were men $(\mathrm{n}=26), 51 \%$ were never smokers $(\mathrm{n}=27)$, and $49 \%$ were smokers $(\mathrm{n}=26)$. Most patients had early-stage disease, including $57 \%(\mathrm{n}=30)$ stage I, 9\% $(\mathrm{n}=5)$ stage II, 32\% ( $\mathrm{n}=17)$ stage III, and $2 \%(\mathrm{n}=1)$ were stage IV.

positive with both PD-L1 TPS of 1\%-49\%. Three patients were checked for $A L K$ gene rearrangement status by fluorescence in situ hybridization, and all were $A L K$ negative.

\section{PD-L1 status}

A total of 163 patients (51\%) had PD-L1-positive tumors, including $24(8 \%)$ with TPS $\geq 50 \%$ and $139(44 \%)$ with TPS of 1\%-49\%. Similar to previous results [9], higher rates of PD-L1 positivity were observed among men (vs. females; chi-square $\mathrm{p}=0.008$ ), smokers (vs. never smokers; chi-square $\mathrm{p}=0.011$ ), and patients with more advanced disease (stages II/ III/IV vs. $\mathrm{I}$; chi-square $\mathrm{p}=0.006$ ) (Table 1). PD-L1 positivity was less common in patients with exon 19 deletion (76 of $145,52 \%$ ) or exon 21 L858R mutation (47 of 121, 39\%) compared with other EGFR mutations ( 40 of $53 ; 75 \%$; chi-square $\mathrm{p}=0.001$ ) (Table 1 ), which include missense mutations in exon 18 ( $n=12)$, insertion, deletion, and missense mutations exon $20(\mathrm{n}=8)$, other mutations in exon 19 (except the deletion mutation in exon 19, $\mathrm{n}=26$ ) and exon 21 (except the L858R mutation $\mathrm{n}=3$ ), and others $(n=4)$. No further subgroup analysis was performed because of the limited sample sizes.

\section{Relationship between PD-L1 expression and RFS and OS}

During a median follow-up of 83 months, 128 disease recurrences and 51 deaths occurred. RFS was significantly shorter ( $\mathrm{p}<0.001, \log$-rank test) in the PD-L1 TPS $\geq 50 \%$ (median, 17.36 months; $95 \%$ confidence interval [CI], 13.9 to 42.7 ) and PD-L1 TPS 1\%-49\% groups (median, 31.6 months; 95\% CI, 28.1 to 39.0) compared with the PD-L1-negative group (median, 35.5 months; 95\% CI, 35.0 to 55.9) (Fig. 2). Using adjusted Cox model 1 and compared with the PD-L1-negative group, the adjusted hazard ratio (HR) for RFS was 1.60 (95\% CI, 0.88 to 2.93) for those with PD-L1 TPS $\geq 50 \%, 1.49$ (95\% CI, 1.02 to 2.18) for those with PD-L1 TPS 1\%-49\%, and 1.51 (95\% CI, 1.05 to 2.18 ) for the combined PD-L1-positive groups (TPS $\geq 1 \%$ ) (Table 2). The results were similar after adjusting for adjuvant 


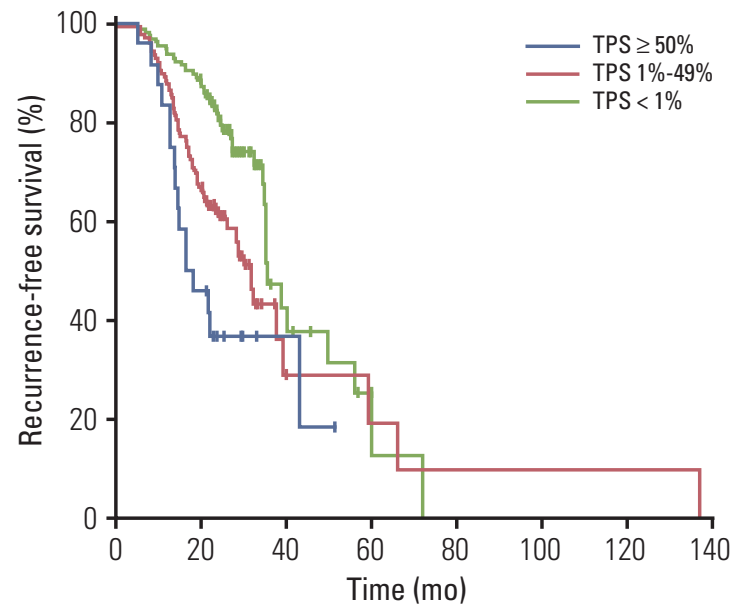

No. at risk

$\begin{array}{rrrrrrrrr}\text { TPS } \geq 50 \% & 24 & 11 & 2 & 0 & 0 & 0 & 0 & 0 \\ \text { TPS } 1 \%-49 \% & 139 & 93 & 3 & 2 & 1 & 1 & 1 & 0 \\ \text { TPS }<1 \% & 156 & 136 & 9 & 1 & 0 & 0 & 0 & 0\end{array}$

Fig. 2. Recurrence-free survival among patients with nonsmall cell lung cancer and epidermal growth factor receptor mutation, by programmed death ligand 1 status. TPS, tumor proportion score.

chemotherapy or radiotherapy (Cox model 2), with an adjusted HR of 1.52 (95\% CI, 0.81 to 2.84) for those with PD-L1 TPS $\geq 50 \%, 1.51$ (95\% CI, 1.02 to 2.21) for those with PD-L1 TPS 1\%-49\%, and 1.51 (95\% CI, 1.04 to 2.19) for the combined PD-L1-positive groups (Table 2).
PD-L1 TPS $\geq 50 \%$ was associated with shorter OS ( $p=0.077$, log-rank test) in the crude analysis (Table 2, Fig. 3), with a crude HR of 2.70 (95\% CI, 1.07 to 6.66) for PD-L1 TPS $\geq 50 \%$ (median OS, 64.5 months; 95\% CI, 34 to not reached) and 1.04 (95\% CI, 0.57 to 1.89) for PD-L1 TPS 1\%-49\% (median OS, 87.8 months; $95 \%$ CI, 66.6 to 123.9), when compared with the PD-L1-negative group (median OS, 76.6 months; 95\% CI, 68.6 to 89.5 ). However, the association was not statistically significant in either adjusted Cox model 1 or 2 (Table 2).

\section{Discussion}

In the current study, which included predominantly patients with early-stage EGFR-mutant NSCLC, $51 \%$ of patients had PD-L1-positive tumors, suggesting that PD-L1 is commonly expressed even among patients with EGFRmutant early-stage NSCLC. Consistent with our previous study [23], a higher prevalence of PD-L1 positivity was observed among men, smokers, and patients with advanced disease. Patients with EGFR exon 19 deletion or exon 21 L858R mutation were less likely to have PD-L1-positive tumors than patients with other EGFR mutations, suggesting that PD-L1 expression may be associated with different types of EGFR mutation status. The exact reason for the association between PD-L1 expression and different types of EGFR mutations is not clear, although both PD-L1 expression and types of EGFR mutations are associated with clinical characteristics such as sex, smoking status, and disease stage [24-26].

Table 2. Cox proportional hazard model for RFS and OS ${ }^{a)}$

\begin{tabular}{|c|c|c|}
\hline \multirow{2}{*}{ Survival } & \multicolumn{2}{|c|}{ HR $(95 \%$ CI $)$} \\
\hline & PD-L1 TPS $\geq 50 \%(n=24)$ & PD-L1 TPS 1\%-49\% (n=139) \\
\hline \multicolumn{3}{|l|}{ RFS } \\
\hline Unadjusted HR (95\% CI) & $3.07(1.74-5.42)$ & $1.89(1.31-2.74)$ \\
\hline Adjusted for baseline prognostic factors ${ }^{\mathrm{b})}$ & $1.60(0.88-2.93)$ & $1.49(1.02-2.18)$ \\
\hline Adjusted for baseline prognostic factors plus treatment ${ }^{c}$ & $1.52(0.81-2.84)$ & $1.51(1.02-2.21)$ \\
\hline \multicolumn{3}{|l|}{ OS } \\
\hline Unadjusted HR (95\% CI) & $2.70(1.07-6.66)$ & $1.04(0.57-1.89)$ \\
\hline Adjusted for baseline prognostic factors ${ }^{\mathrm{b})}$ & $1.25(0.46-3.44)$ & $0.94(0.5-1.76)$ \\
\hline Adjusted for baseline prognostic factors plus treatment ${ }^{c}$ & $1.29(0.45-3.72)$ & $0.93(0.49-1.74)$ \\
\hline
\end{tabular}

RFS, recurrence-free survival; OS, overall survival; HR, hazard ratio; CI, confidence interval; PD-L1, programmed death ligand 1 ; TPS, tumor proportion score. a)PD-L1 negative was used as the reference population $(\mathrm{n}=156)$, b)Baseline prognostic factors included age, sex, smoking status, stage, and Eastern Cooperative Oncology Group performance status (Cox model 1), cIncluded baseline prognostic factors and treatment (Cox model 2). 


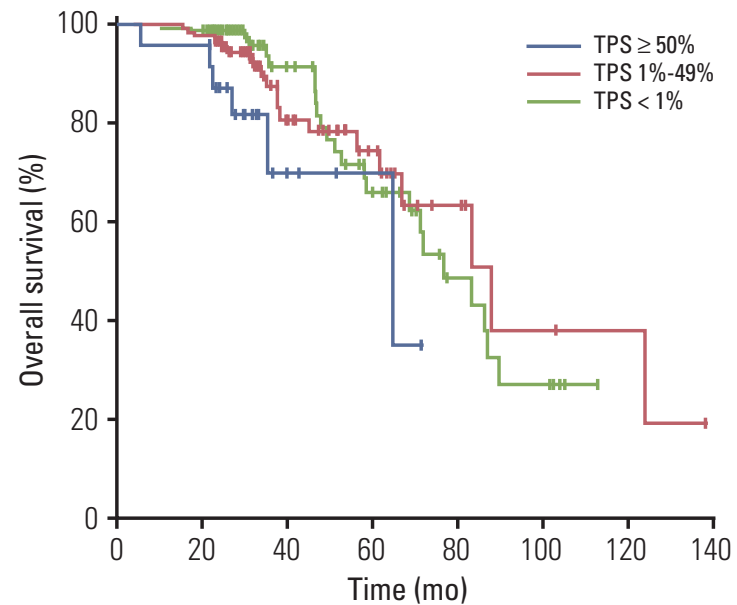

No. at risk

$\begin{array}{rrrrrrrrr}\text { TPS } \geq 50 \% & 24 & 23 & 4 & 2 & 0 & 0 & 0 & 0 \\ \text { TPS } 1 \%-49 \% & 139 & 136 & 34 & 17 & 7 & 3 & 2 & 0 \\ \text { TPS }<1 \% & 156 & 152 & 39 & 22 & 9 & 5 & 0 & 0\end{array}$

Fig. 3. Overall survival among patients with non-small cell lung cancer and epidermal growth factor receptor mutation, by programmed death ligand 1 status. TPS, tumor proportion score.

At this point, data are limited regarding the prognostic effect of PD-L1 among patients with EGFR mutations. The correlation between tumor PD-L1 status and EGFR mutation status has been studied previously, with contradictory results [24-26]. One study showed a higher EGFR mutation rate in patients with lower PD-L1 expression and that the presence of EGFR mutation increased OS, whereas high PD-L1 expression decreased OS [24]. However, this study did not explore the relationship of uncommon EGFR mutations. Rather, it focused on the effect of the presence or absence of EGFR mutations and PD-L1 expression [24]. Another study showed that there was no significant relationship between PD-L1 expression, common baseline characteristics, and EGFR mutation, but there was an association of PD-L1 expression with response to EGFR TKI [26]. Furthermore, in the group of patients with EGFR mutations, PFS and OS of patients with PD-L1-positive tumors tended to be longer than in patients with PD-L1-negative tumors; however, statistical significance was not achieved [26]. This may have been due to the smaller sample size $(n=170)$ in the Tang et al.'s study [26] compared with the current study.

It has been postulated that EGFR activation promotes expression of PD-L1 by the nuclear factor $\mathrm{\kappa B}$ pathway [27]. This pathway may underlie acquired resistance to EGFR TKIs [27]. Thus, combined targeted therapy using immune checkpoint inhibitors and EGFR TKIs may provide a novel therapeutic option for patients with EGFR-mutant NSCLC [27]. Preliminary results from the CheckMate 012 study in patients with advanced NSCLC reported that nivolumab 3 $\mathrm{mg} / \mathrm{kg}$ intravenously every 2 weeks and erlotinib $150 \mathrm{mg}$ orally daily enabled $15 \%$ of TKI-refractory patients to achieve a partial response (3 of 20) [28]. Ongoing clinical trials that are investigating the combination of immune checkpoint inhibitor and EGFR TKI include a phase I study investigating the recommended phase II dose of the immune checkpoint inhibitors nivolumab or ipilimumab and erlotinib or crizotinib in patients with advanced NSCLC and EGFR mutations or ALK translocations (no longer enrolling; ClinicalTrials.gov identifier, NCT01998126) and a phase I study of pembrolizumab and afatinib in patients with advanced NSCLC (currently enrolling; ClinicalTrials.gov identifier, NCT02364609).

The prognostic implications of PD-L1 expression and OS have been inconsistent, with studies or meta-analyses providing negative and positive associations $[9,29,30]$. In the current study, we observed a trend of negative association between PD-L1 expression and OS, particularly in the subgroup of patients with TPS $\geq 50 \%$; however, the result was not statistically significant after adjusting for other baseline characteristics. Part of the reason is because of the good prognosis of this patient population (e.g., patients with EGFRmutant, early-stage adenocarcinoma) and relatively shorter follow-up, and only 51 deaths were observed during the median follow-up of 83 months. Our study suggests that positive PD-L1 expression may be associated with short RFS.

The strengths of this study include a homogeneous population of patients with NSCLC and EGFR mutations, relatively large sample size, and complete clinical and outcome information. In addition, we used the FDA-approved companion diagnostic immunohistochemical assay (PD-L1 IHC 22C3 pharmDx), which is the same as that used in pembrolizumab clinical trials. Limitations to this study include the retrospective nature of the analysis, small number of patients with stage IV disease, and lack of comparison with patients with wild-type EGFR. In addition, $97 \%$ of the patients had adenocarcinoma, and we were not able to examine the association between PD-L1 expression and survival among other histologic subtypes of patients.

In conclusion, our results suggest that PD-L1 is commonly expressed among patients with early-stage NSCLC with EGFR mutations, and higher PD-L1 expression may be associated with short RFS for patients with EGFR-mutant NSCLC. Notably, the type of EGFR mutation may affect the expression of PD-L1, although this does not appear to have an effect on survival. The relationship of these results with response to currently available therapies targeted to EGFR or PD-1/ PD-L1 remains to be investigated. 


\section{Conflicts of Interest}

This study was supported by Merck \& Co., Inc. (Kenilworth, NJ, USA). Editorial assistance was provided by Jennifer M. Kulak, PhD, of the ApotheCom oncology team (Yardley, PA, USA) and was funded by Merck \& Co., Inc., Kenilworth, NJ, USA. Drs. Cho, Y.-L. Choi, Sun, H. Choi, T.-E. Kim, and J. Kim have no conflicts to disclose. Drs Zhou, Dolled-Filhart, Emancipator, and Rutkowski are employees of Merck Sharp \& Dohme Corp., a subsidiary of Merck \&
Co., Inc., Kenilworth, NJ, USA and own stock in the company. Dr. Emancipator also has stock in Bayer AG and Johnson and Johnson and his spouse is employed by and has stock ownership in Celgene.

\section{Acknowledgments}

The authors thank Eric Rubin, Gregory M. Lubiniecki, Cong Chen, Jared Lunceford, Jun Hun, Melissa Whipple, and Qing Shao (all of Merck and Co., Inc., Kenilworth, NJ, USA).

\section{References}

1. D'Addario G, Fruh M, Reck M, Baumann P, Klepetko W, Felip E, et al. Metastatic non-small-cell lung cancer: ESMO Clinical Practice Guidelines for diagnosis, treatment and follow-up. Ann Oncol. 2010;21 Suppl 5:v116-9.

2. Shigematsu H, Lin L, Takahashi T, Nomura M, Suzuki M, Wistuba II, et al. Clinical and biological features associated with epidermal growth factor receptor gene mutations in lung cancers. J Natl Cancer Inst. 2005;97:339-46.

3. Bronte G, Rizzo S, La Paglia L, Adamo V, Siragusa S, Ficorella $\mathrm{C}$, et al. Driver mutations and differential sensitivity to targeted therapies: a new approach to the treatment of lung adenocarcinoma. Cancer Treat Rev. 2010;36 Suppl 3:S21-9.

4. Luo SY, Lam DC. Oncogenic driver mutations in lung cancer. Transl Respir Med. 2013;1:6.

5. Shi Y, Au JS, Thongprasert S, Srinivasan S, Tsai CM, Khoa MT, et al. A prospective, molecular epidemiology study of EGFR mutations in Asian patients with advanced non-small-cell lung cancer of adenocarcinoma histology (PIONEER). J Thorac Oncol. 2014;9:154-62.

6. McDermott DF, Atkins MB. PD-1 as a potential target in cancer therapy. Cancer Med. 2013;2:662-73.

7. D’Incecco A, Andreozzi M, Ludovini V, Rossi E, Capodanno A, Landi L, et al. PD-1 and PD-L1 expression in molecularly selected non-small-cell lung cancer patients. Br J Cancer. 2015;112:95-102.

8. Zou W, Chen L. Inhibitory B7-family molecules in the tumour microenvironment. Nat Rev Immunol. 2008;8:467-77.

9. Sun JM, Zhou W, Choi YL, Choi SJ, Kim SE, Wang Z, et al. Prognostic significance of PD-L1 in patients with non-small cell lung cancer: a large cohort study of surgically resected cases. J Thorac Oncol. 2016;11:1003-11.

10. Azuma K, Ota K, Kawahara A, Hattori S, Iwama E, Harada T, et al. Association of PD-L1 overexpression with activating EGFR mutations in surgically resected nonsmall-cell lung cancer. Ann Oncol. 2014;25:1935-40.

11. Sul J, Blumenthal GM, Jiang X, He K, Keegan P, Pazdur R. FDA approval summary: pembrolizumab for the treatment of patients with metastatic non-small cell lung cancer whose tumors express programmed death-ligand 1. Oncologist. 2016;21:643-50.
12. Kazandjian D, Suzman DL, Blumenthal G, Mushti S, He K, Libeg $\mathrm{M}$, et al. FDA approval summary: nivolumab for the treatment of metastatic non-small cell lung cancer with progression on or after platinum-based chemotherapy. Oncologist. 2016;21:634-42.

13. Genentech, Inc. Tecentriq [package insert]. South San Fransisco, CA: Genentech, Inc.; 2016.

14. Boland JM, Kwon ED, Harrington SM, Wampfler JA, Tang H, Yang $\mathrm{P}$, et al. Tumor B7-H1 and B7-H3 expression in squamous cell carcinoma of the lung. Clin Lung Cancer. 2013;14: 157-63.

15. Chen YB, Mu CY, Huang JA. Clinical significance of programmed death-1 ligand-1 expression in patients with nonsmall cell lung cancer: a 5-year-follow-up study. Tumori. 2012;98:751-5.

16. Mu CY, Huang JA, Chen Y, Chen C, Zhang XG. High expression of PD-L1 in lung cancer may contribute to poor prognosis and tumor cells immune escape through suppressing tumor infiltrating dendritic cells maturation. Med Oncol. 2011;28: 682-8.

17. Yang CY, Lin MW, Chang YL, Wu CT, Yang PC. Programmed cell death-ligand 1 expression in surgically resected stage I pulmonary adenocarcinoma and its correlation with driver mutations and clinical outcomes. Eur J Cancer. 2014;50: 1361-9.

18. Dolled-Filhart M, Roach C, Toland G, Stanforth D, Jansson M, Lubiniecki GM, et al. Development of a companion diagnostic for pembrolizumab in non-small cell lung cancer using immunohistochemistry for programmed death ligand-1. Arch Pathol Lab Med. 2016 Aug 23 [Epub]. https:// doi.org/10.5858 / arpa.2015-0542-OA.

19. Roach C, Zhang N, Corigliano E, Jansson M, Toland G, Ponto $\mathrm{G}$, et al. Development of a companion diagnostic PD-L1 immunohistochemistry assay for pembrolizumab therapy in non-small-cell lung cancer. Appl Immunohistochem Mol Morphol. 2016;24:392-7.

20. Edge S, Byrd DR, Compton CC, Fritz AG, Greene FL, Trotti A. AJCC cancer staging manual. 7th ed. New York: Springer-Verlag; 2010.

21. Garon EB, Rizvi NA, Hui R, Leighl N, Balmanoukian AS, Eder 
JP, et al. Pembrolizumab for the treatment of non-small-cell lung cancer. N Engl J Med. 2015;372:2018-28.

22. Herbst RS, Baas P, Kim DW, Felip E, Perez-Gracia JL, Han JY, et al. Pembrolizumab versus docetaxel for previously treated, PD-L1-positive, advanced non-small-cell lung cancer (KEYNOTE-010): a randomised controlled trial. Lancet. 2016;387: 1540-50.

23. Sun JM, Zhou W, Choi YL, Choi SJ, Kim SE, Wang Z, et al. PD-L1 expression and survival in patients with non-small cell lung cancer (NSCLC) in Korea. J Clin Oncol. 2014;32 Suppl 5:Abstr 8066.

24. Ji M, Liu Y, Li Q, Li X, Ning Z, Zhao W, et al. PD-1/PD-L1 expression in non-small-cell lung cancer and its correlation with EGFR/KRAS mutations. Cancer Biol Ther. 2016;17: 407-13.

25. Ji M, Liu Y, Li Q, Li XD, Zhao WQ, Zhang H, et al. PD-1/ PD-L1 pathway in non-small-cell lung cancer and its relation with EGFR mutation. J Transl Med. 2015;13:5.

26. Tang Y, Fang W, Zhang Y, Hong S, Kang S, Yan Y, et al. The association between PD-L1 and EGFR status and the prognostic value of PD-L1 in advanced non-small cell lung cancer patients treated with EGFR-TKIs. Oncotarget. 2015;6:14209-19.

27. Lin K, Cheng J, Yang T, Li Y, Zhu B. EGFR-TKI down-regulates PD-L1 in EGFR mutant NSCLC through inhibiting NF-kB. Biochem Biophys Res Commun. 2015;463:95-101.

28. Rizvi NA, Chow LQ, Borghaei H, Shen Y, Harbison C, Alaparthy $S$, et al. Safety and response with nivolumab (antiPD-1; BMS-936558, ONO-4538) plus erlotinib in patients (pts) with epidermal growth factor receptor mutant (EGFR MT) advanced NSCLC. J Clin Oncol. 2014;32 Suppl 5:Abstr 8022.

29. Sorensen SF, Zhou W, Dolled-Filhart M, Georgsen JB, Wang Z, Emancipator K, et al. PD-L1 expression and survival among patients with advanced non-small cell lung cancer treated with chemotherapy. Transl Oncol. 2016;9:64-9.

30. Schmidt LH, Kummel A, Gorlich D, Mohr M, Brockling S, Mikesch JH, et al. PD-1 and PD-L1 expression in NSCLC indicate a favorable prognosis in defined subgroups. PLoS One. 2015;10:e0136023. 